\title{
INVERSE FILTERING METHOD FOR SUPER-RESOLUTION DIGITAL IMAGING
}

\author{
Ratko Ivković ${ }^{1,3, *}$, \\ Mile Petrović1, \\ Ivana Milošević ${ }^{2}$, \\ Dejan Đukić3, \\ Vladimir Maksimović1 \\ 'Faculty of Technical Sciences, \\ University of Pristina, \\ Kosovska Mitrovica, Serbia \\ 2SP Audio and Video Technologies, \\ The School of Electrical and Computer \\ Engineering, \\ Belgrade, Serbia \\ ${ }^{3}$ Faculty of Information Technology, \\ Alfa BK University, \\ Belgrade, Serbia
}

\begin{abstract}
:
Super-resolution is a method of combining various techniques in digital image processing, in order to achieve higher resolution in certain image segments, which initially had insufficient resolution and quality. The principal idea in this work is the use of the analysis of the level of detail and of the standard deviation, which results in enhancing the resolution of the digital image, whilst maintaining the overall image quality. In the first part of this article, an experimental method for estimating the standard deviation and the level of detail in a digital image has been shown, together with the inferred facts that result from this analysis. The second part of this article uses these facts in the process of designing an inverse filter by applying the averages of pixel intensities. The proposed method has significant advantages over the heretofore used super-resolution techniques, as it achieves the enhancement of the resolution from a single image, whilst retaining the initial image potential.
\end{abstract}

Keywords:

Digital Image Processing, Super-Resolution, Inverse Filtering, Segments, Matrix.

\section{INTRODUCTION}

Resolution of an image describes the number of details that can be shown in this image. Resolution of a digital image is limited by the used digital image recording equipment such as the optics, the sensors (CCD, CMOS), or the electronics. The price of the equipment for producing high resolution digital images is extremely high, and it is prohibitive in the vast majority of imaging applications. On the other hand, it may be possible to use certain image processing algorithms in order to achieve the required enhancement in the image quality. Moreover, in some cases, even the digital images recorded using an expensive imaging equipment may be further enhanced by using some of those algorithms. All of the algorithms and their combinations $[1,2]$ that aim directly at the image resolution enhancement are summarily called the Super-Resolution (SR) methods [3, 4]. Super-Resolution algorithms have been the subject of scientific interest since the inception of the digital image processing, and up until today. The main areas of the application of the S-R:

- Remote sensing - S-R achieves correct interpretation of remotely taken images of areas and regions that may be harmful for humans. 
- Surveillance imaging and video - Surveillance cameras usually cover large areas, which implies that it is not possible to obtain high resolution imaging over the totality of the area under surveillance.

- Medical imaging - Usually, there are multiple images, taken using different methods and equipment. Hence, there is often a need for the application of S-R algorithms in order to obtain a correct interpretation.

- Image and video conversion - Methods for image and video format conversion often use some of $\mathrm{S}$-R methods.

The principal aim of S-R methods is to obtain an image with enhanced resolution from one or multiple low-resolution images. There are two main kinds of S-R methods:

1. Classical S-R methods, or S-R methods based on the processing of multiple images (nowadays often seen in modern mobile handsets), and

2. Single image S-R methods.

Classical S-R methods (see $[5,6,7]$ ) use multiple images of the same scene. Each of these images is normally of limited quality and resolution, sometimes with unknown values of the image quality parameters. From these images, an image of high quality and enhanced resolution needs to be reconstructed. Classical S-R methods are mainly based on sub-pixel clustering. Single image S-R methods have had limited success in digital image resolution enhancement (see [8] and [9]). As may be seen in $[10,11,12,13,14,15,16,17]$, there are multiple ways to achieve resolution enhancement. In this article, the main idea is to design a resolution enhancement (S-R) algorithm by inverting the averaging filtering methods (blurring, median, or mean value filters), or the noise reduction filtering methods. By investigating image segments with visually similar colouring, it may be observed that the pixel values in these segments are not greatly different (see Figs. 1a, 1b, and 1c). The segment under observation in the image is marked by a circle, and the corresponding pixel values are shown in the top right corner of the figure. Although the selected segments are visually very similar, it is evident that the deviations in the pixel values in any of the three colour channels $(R, G, B)$ within the marked segments may be up to $23 \%$ of the range of the values (0-255).

As has been seen in Fig. 1a, 1b, and 1c, pixel values in the same channel differ from each other. E.G. the selected pixel in Fig. 1b has RGB colour values (201,
88, 90), whereas the selected pixel in Fig. 1c has values $(194,69,73)$. Within the selected segments in Fig. 1, there are no details, so it is not possible to consider the level of detail. However, it is reasonable to estimate the standard deviation for the image regions with visually similar uniform colour. Therefore, the principal idea for designing the S-R method presented in this article has been the variability in pixel values in the neighbourhood of the selected pixel.

a)

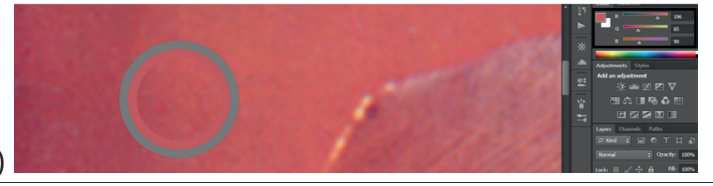

b)

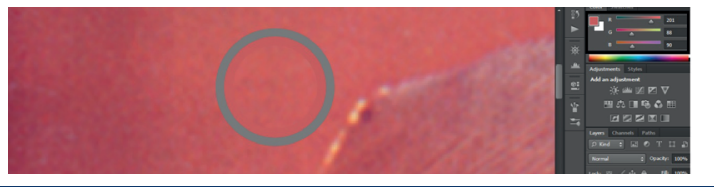

c)

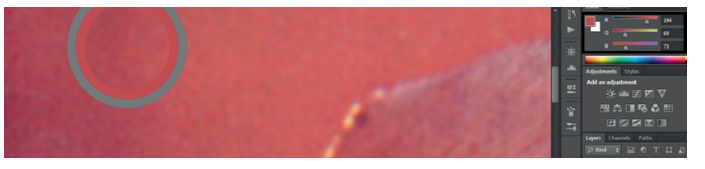

Fig. 1. Three regions selected within a single image segment

Three regions selected within a single image segment

So far, the methods for generating an image with enhanced resolution generally required the use of multiple low-resolution images. However, the algorithm proposed in this article uses only one low resolution image as the only source of data for analysis, and subsequently for creating an enhanced resolution image. Generating an image with enhanced resolution from images with low resolution may be viewed as the inverse of the process of image resolution reduction. Hence, the S- $R$ method proposed in this article is being denoted an inverse filtering method.

\section{THEORETICAL FRAMEWORK}

The method proposed in this article is effectuated in two phases. In the first phase, the standard deviation of pixel values and the level of detail in the original lowresolution image are being estimated. In this process, it is not necessary to analyse the standard deviation using the whole image, as, in this estimate, the compression errors, image textures, edges, and noise would be taken into account, whilst these image details and artefacts, as 
a rule, would have greatly different statistical descriptions. E.G. image segments that are preponderately red and that are preponderately blue, when taken together would have excessively large values of standard deviation. Therefore, the results of the analysis of standard deviation of pixel value distribution and of the level of detail in the whole image would not be valid. Thus, before the analysis of the standard deviation and of the level of detail, the image needs to be appropriately segmented into segments with approximately uniform colour. Then, for each segment, the standard deviation and the level of detail are being estimated. Further on, the estimation of image parameters is performed separately for each of the three colour channels: red, green, and blue. The estimated values obtained in this way are then being compared. As it has been shown in [18], the greatest value of the estimated standard deviation corresponds to the greatest level of image detail, that is, the greater the variability in the image, the greater the level of detail. This fact is being used in the S-R method described in this article. A problem may appear here as to how to segregate image segments with near but not quite the same colour values. The reasoning in this case leads to the use of the analysis of the presence of the edges in the image. Since edge is defined as the image coordinate at which there is an abrupt change of some of the image parameters (in this case, of the colour), the image regions surrounded by edges are considered to have pixels of the sufficiently similar values for the application of the proposed S-R method.

\section{METHODOLOGICAL FRAMEWORK}

Based on the theoretical framework described in the previous section, by which the standard deviation of the pixel values is clearly related to the level of image details, the segmented image will be used as a skeleton of the $\mathrm{S}-\mathrm{R}$ enhanced image. It is therefore necessary firstly to detect the details in the original image, and then to estimate the standard deviation separately for each of the determined image segments.

The resolution enhancement is then performed as the image reconstruction by applying the inverted form of averaging filters. E.G. a 3 by 3 pixel region of the image has pixel values noted by Y1, Y2... Y9:
Table 1. Pixel values in the observed segment of the original image

\begin{tabular}{ccc}
\hline Y1 & Y2 & Y3 \\
\hline Y4 & Y5 & Y6 \\
\hline Y7 & Y8 & Y9 \\
\hline
\end{tabular}

The filter for image reconstruction is then defined by applying the following filter:

Table 2. Pixel values of the observed image segment of the reconstructed image

\begin{tabular}{ccl}
\hline $\mathrm{StD}(-\mathrm{x}, \mathrm{x})+\mathrm{Y} 1$ & $\mathrm{StD}(-\mathrm{x}, \mathrm{x})+\mathrm{Y} 1$ & $\mathrm{StD}(-\mathrm{x}, \mathrm{x})+\mathrm{Y} 1$ \\
\hline $\mathrm{StD}(-\mathrm{x}, \mathrm{x})+\mathrm{Y} 1$ & $\mathrm{Y} 1$ & $\mathrm{StD}(-\mathrm{x}, \mathrm{x})+\mathrm{Y} 1$ \\
\hline $\mathrm{StD}(-\mathrm{x}, \mathrm{x})+\mathrm{Y} 1$ & $\mathrm{StD}(-\mathrm{x}, \mathrm{x})+\mathrm{Y} 1$ & $\mathrm{StD}(-\mathrm{x}, \mathrm{x})+\mathrm{Y} 1$ \\
\hline
\end{tabular}

where $\operatorname{StD}(-x, x)$ is the standard deviation of the pixel values in the image segment being processed.

In the neighbourhood of pixel Y1, there are pixels that, statistically, have values within range from Y1 - StD to $\mathrm{Y} 1+\mathrm{StD}$. In this way, a filter is defined, that performs an operation which is the inverse of the pixel averaging in 3 by 3 subimages. Practically, the inverse averaging filter is designed by adding randomly generated values from the interval from $-\mathrm{StD}$ to $+\mathrm{StD}$ to the neighbouring pixels to pixel Y1. Pixel Y1 is thus being surrounded by 8 new values, as it has been shown in Table 2 .

E.G. if StD is 5, the values added to the neighbouring pixels are generated randomly in the interval $(-5$, $+5)$. If the value of pixel Y1 is 100 , then the neighbouring pixels will obtain values from the interval $(95,105)$. These values are not necessarily uniformly distributed, but will match the pixel value distribution of the original pixels in the image. In order to reduce drastic effects that may appear by performing the filtering near the segment edges, a mild form of weighted filter is being used here $[19,20]$.

\section{RESULTS AND DISCUSSION}

The proposed S-R method has been tried on 30 digital images, and also separately on 22 image segments, for the purposes of testing the method. Some of these images have been the images of physical objects and recorded using optical and image sensing equipment, whilst others have been synthetic images that have been generated by the computer. The results of the analysis of standard deviations have been presented graphically in Fig. 2. 
StD

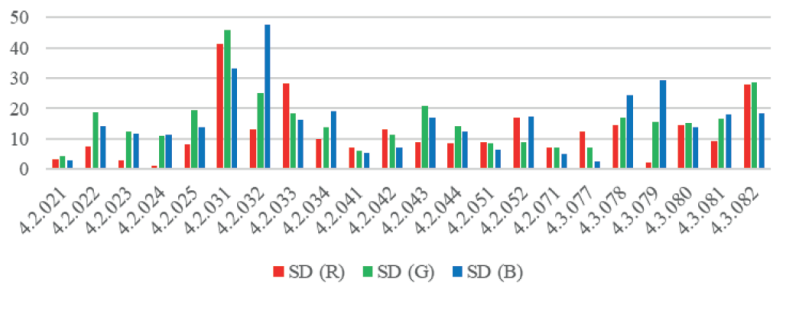

Fig. 2. Standard deviation of the image segments under investigation

Although the estimated standard deviation is not considered as a measure of image quality, it has been used as an indicator of pixel value variation of the neighbouring pixels. Here it needs to be underlined that there is not a single value of standard deviation in a segment, but there are three separate values, one for each colour channel. A justification of this approach is clearly visible in the results of standard deviation estimation for segments 4.2.32 and 4.3.079, in which there is a significant difference between the three estimates of the standard deviation. Such cases of different estimates of standard deviation in colour channels will be further compared with the estimate of the level of detail, in order to determine the quality of that digital image.

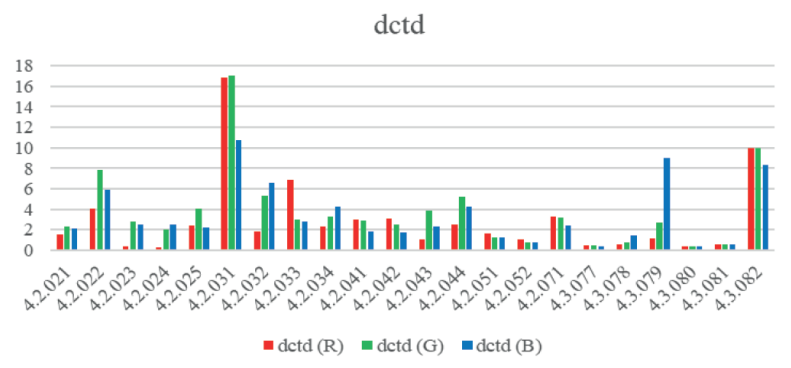

$\overline{\text { Fig. 3. Level of detail in the segments under investigation, }}$ obtained from the DCT

$\mathrm{StD} / \mathrm{dctd}$

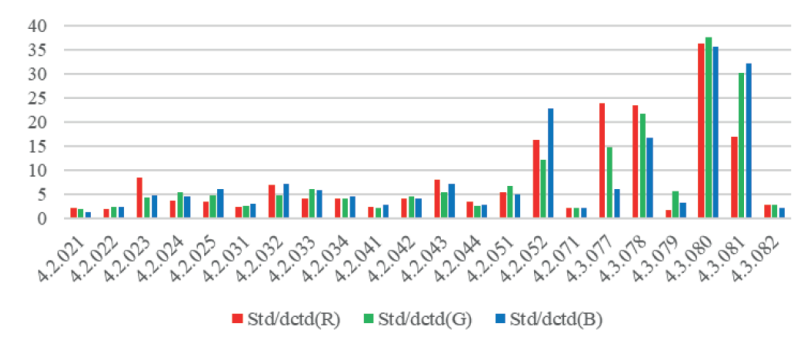

Fig. 4. Ratio between the estimated standard deviation and the level of details
As it has been shown in [18], the level of detail is not strongly correlated with the estimated standard deviation of the pixel values. Nevertheless, these two parameters show similar behaviour, except in case of images corrupted by the presence of noise. This observation is of utmost importance, as it offers a definite unique criterion as to which segments, and ultimately which images, are amenable to treatment using the S-R method described here. A convenient method for comparing these two image parameters is by computing the ratio between them. The conclusions of the observations presented in [21,22] are further corroborated by observing the corresponding oscillations of the two parameters describing the image quality separately in each of the colour channels.

Despite the fact that the variations of these two parameters in an image segment may have high values in each of the colour channels, their ratio remains approximately constant, when the level of noise in the image is low [23]. However, as may be seen in segments 4.3.077 and 4.3.081, significant differences between these ratios indicate the existence of a high level of noise. It has been determined experimentally that the limiting level of noise for this S-R method is about $11 \%$. In cases of images with higher level of noise, the results produced with this method deteriorate. When the level of noise is above $15 \%$, and when the original image is of low resolution, the image resolution enhancement should be attempted by using one of the classical S-R methods, based on processing multiple images of the same scene. Inverse filtering method for image reconstruction may achieve the resolution enhancement up to three times per linear dimension, both horizontally and vertically, which has been shown in Fig. 5. Further assessment of the quality of the reconstructed image may be obtained by comparing the estimated level of details in these two images. Significant increase of the level of details in all of the segments of the reconstructed image is presented in Fig. 7. However, low level of details in images with initially low-resolution is a prohibiting condition for the presence of details in the corresponding reconstructed images. The quality of the reconstructed image when juxtaposed to the original image may be seen in Fig. 6, in which the original and the reconstructed images have been shown at the same resolution. 


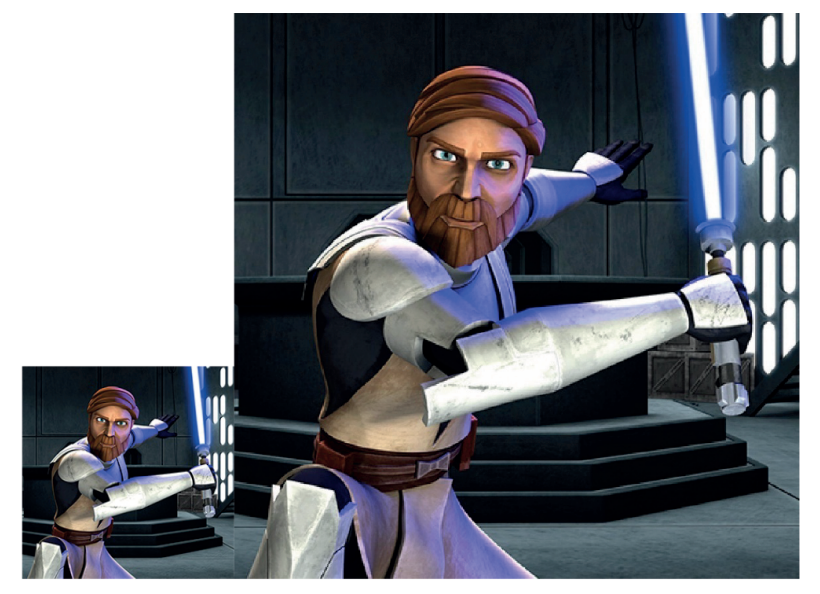

Fig. 5. Image reconstruction and resolution enhancement by using inverse filtering

a)
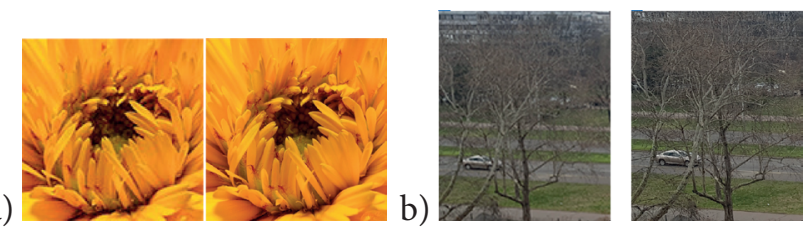

c)

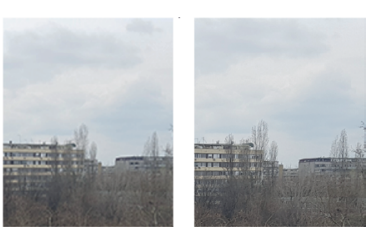

Fig. 6. Comparison of the original images and the reconstructed images with increased resolution obtained by using inverse filtering

(Note: images shown here have identical resolution)

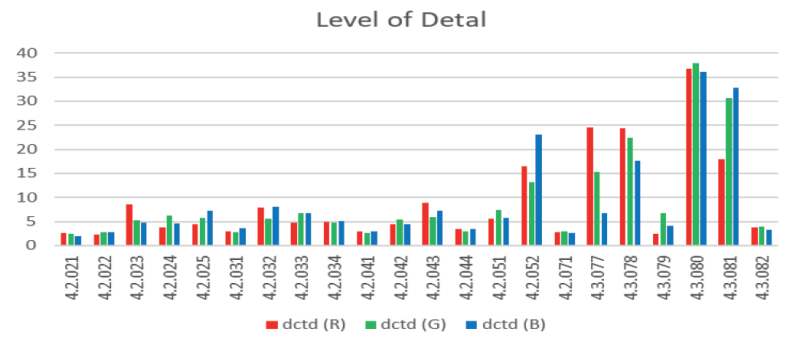

Fig. 7. Level of detail in reconstructed digital images

As has been shown in Fig. 7, relative increase of the level of detail in reconstructed images may attain high values, up to $46 \%$ for segment 4.3.082. However, by comparing this increase with the values presented in Fig. 3. it may be observed that the increase in the level of details is the greatest in images which initially have low level of details. This is a beneficial property of the algorithm described here, as the images with a low level of details are perceived as visually most appealing. An example of this has been shown in Fig. 6. Standard deviation estimates in image segments in Fig $6 \mathrm{~b}$ and $6 \mathrm{c}$ have values of up to 137 , that is more than half of the available range (0-255). Therefore, each of these segments requires a different processing approach. Such high values of standard deviation are usually present in images of trees or buildings, but it is not the case with image segments with sky, or lawns, especially when these surfaces are sufficiently distant from the objective of the image recording camera.

Filtering images with typographic content, i.e. with black characters on white background, is an area of great interest in image processing. This type of images is characterised by low level of details, and by high contrast and clear transition between the objects, i.e. characters, letters, and the background i.e. the white surface (Fig. 9a). It has been considered that the images of this kind are most suitable for being processed by some of S-R methods. On the other hand, in the images with low resolution the transitions are not very clear, and a great loss of detail and contrast may be observed. Yet, it is exactly with the images of this kind that the S-R method described here attains a very high level of quality of reconstructed images. Application of the algorithm as described in section Methodological framework, i.e. with estimating standard deviation separately for each colour channel, and then generating 3 by 3 matrices with new pixel values, achieves preservation of the original information in the image. The results of comparison of the success of this method with other methods usually applied to this kind of images have been shown in Fig. 9b, $9 \mathrm{c}$, and $9 \mathrm{~d}$, from which it is evident that visually the best reconstructed image is in Fig. 9d. It may be of interest to notice that, in this kind of images, the aim is to reduce the level of details in the image, which is achieved by increasing the sharpness of transition between the objects and the background, and which has been best achieved by the S-R method proposed in this article. 
Incresing in \%

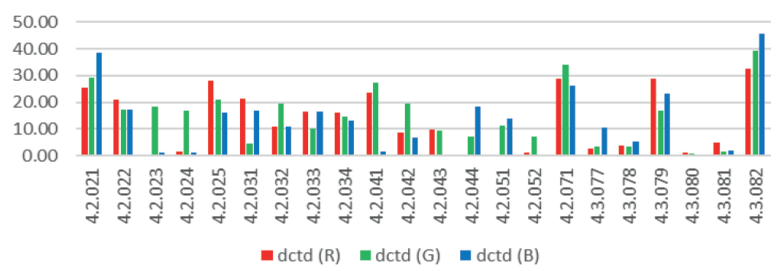

Fig. 8. Relative increase of the level of detail

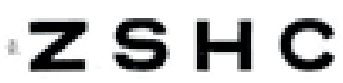

HSKR N CHKRVD HONSDC V OKHDNRCS $\checkmark H D N K U O S R C$ D D CLKZVHB MOA

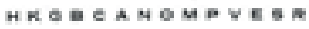

a)

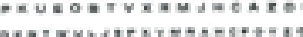

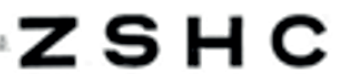
HSKR N CHKRVD HONSDC V OKHDNRCS i $V$ HDNKUOSRC DOCLKZVHB H c)

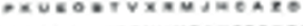

Fig. 9. Comparison of various S-R algorithms: a) original image, b) bicubic interpolation, c) Unified single-image $\mathrm{S}-\mathrm{R}$ and $\mathrm{d}$ ) Inverse filtering method

\section{CONCLUSION}

In this article, a novel algorithmic solution for SuperResolution has been presented. The prominent quality of this solution is that it can reconstruct an enhanced resolution image from only one low resolution image. This solution is based on the analysis of standard deviation and on the weighted filter, and the essence of the method is the analysis of sensitivity of the neighbourhood of an image segment. The method is capable of enhancing the image resolution up to three times per dimension with respect to the original image. The results obtained on test images have demonstrated the effectiveness of the described method, and have shown that it compares favourably when compared to other S-R algorithms. This method has a wide area of application, but excellent results have been obtained by its application to the images with low level of details, such as images of human faces, images generated by computer animation, and, also, good results have been obtained by processing images with typographic content. A promising direction for future research will be in combining this algorithm with image segmentation methods, which will lead to even higher quality of reconstructed images.

\section{ACKNOWLEDGMENT}

This work was done within the research project of the Ministry of Science and Technological Development of Serbia TR35026, III47016 and III44006.

\section{REFERENCES}

[1] R. Tenne, U. Rossman, B. Rephael, Y. Israel, A. Krupinski-Ptaszek, R. Lapkiewicz, Y. Silberberg and Dan Oron, "Super-resolution enhancement by quantum image scanning microscopy," Nature Photonicsvolume, vol. 13, pp. 116-122, 2019.

[2] X. Wang, K. Yu, C. Dong and C. Change Loy, "Recovering Realistic Texture in Image Super-resolution by Deep Spatial Feature Transform," 2018 IEEE/CVF Conference on Computer Vision and Pattern Recognition (CVPR), pp. 1 -23, June 2018.

[3] Y. Zhang, Y. Tian, Y. Kong, B. Zhong and Y. Fu, "Residual Dense Network for Image Super-Resolution," 2018 IEEE/CVF Conference on Computer Vision and Pattern Recognition (CVPR), pp. 2472 - 2481, June 2018.

[4] C. Cruz, R. Mehta, V. Katkovnik and K. O. Egiazarian, "Single Image Super-Resolution Based on Wiener Filter in Similarity Domain," IEEE transactions on image processing, vol. 27, no. 3, pp. 1376-1389, March 2018.

[5] M. Irani and S. Peleg. Improving resolution by image registration. CVGIP, (3), 1991.

[6] D. Capel. Image Mosaicing and Super-Resolution. Springer-Verlag, 2004.

[7] S. Farsiu, M. Robinson, M. Elad, and P. Milanfar. Fast and robust multiframe super resolution. T-IP, (10), 2004

[8] S. Baker and T. Kanade. Limits on super-resolution and how to break them. PAMI, (9), 2002.

[9] Z. Lin and H. Shum, "Fundamental Limits of Reconstruction-Based Superresolution Algorithms under Local Translation," PAMI, vol.1, 2004. 
[10] R. C. Hardie, K. J. Barnard, and E. A. Armstrong, "Joint map registrationand high-resolution image estimation using a sequence of undersampled images," IEEE Transactions on Image Processing, vol. 6, pp. $1621\{1633,1997$.

[11] S. Farsiu, M. D. Robinson, M. Elad, and P. Milanfar, "Fast and robust multiframe super-resolution," IEEE Transactions on Image Processing, vol. 13, pp. 1327-1344, 2004.

[12] M. E. Tipping and C. M. Bishop, "Bayesian image super-resolution," in Advances in Neural Information Processing Systems (NIPS), pp. 13031310,2002 .

[13] S. Baker and T. Kanade, "Limits on super-resolution and how to breakthem," IEEE Transactions on Pattern Analysis and Machine Intelligence, vol. 24, no. 9, pp. 1167-1183, Sep. 2002.

[14] H. S. Hou and H. C. Andrews, "Cubic spline for image interpolation anddigital filtering," IEEE Transactions on Signal Processing, vol. 26, pp. 508-517, 1978.

[15] S. Dai, M. Han, W. Xu, Y. Wu, and Y. Gong, "Soft edge smoothness priorfor alpha channel super resolution," in IEEE Conference on Computer Visionand Pattern Classification (CVPR), pp. 1-8, 2007.

[16] J. Sun, Z. Xu, and H. Shum, "Image super-resolution using gradient profileprior," in IEEE Conference on Computer Vision and Pattern Recognition (CVPR), pp. 1-8, 2008.

[17] W. T. Freeman, E. C. Pasztor, and O. T. Carmichael, "Learning low-levelvision," International Journal of Computer Vision, vol. 40, no. 1, pp. 25-47, 2000.
[18] R. Ivković, I. Milosević, B. Gara, M. Pavlović, D. Miljković, "Analysis of digital image standard deviation and level of detail," INFOTEH 2015, XIV međunarodni naučno-stručni simpozijum, Jahorina, Bosna i Hercegovina, pp. 600-603, 2015.

[19] D. R. K. Brownrigg, "The weighted median filter," Communications of the ACM, vol. 27, no.8, pp. 807-818, 1984.

[20] L. Alparone, M. Barni, F. Bartolini, and V. Cappellini, "Adaptively weighted vector-median filters for motion-fields smoothing," 1996 IEEE International Conference on Acoustics, Speech, and Signal Processing Conference Proceedings, vol. 4, pp. 2267 2270, 1996.

[21] R. Ivković, M. Petrović, D. Miljković, P. Spalević and I. Milošević, "Reduction of Snow and Rain Noise in Spatial Domain," in Sinteza 2016 - International Scientific Conference on ICT and E-Business Related Research, Belgrade, Singidunum University, Serbia, pp. 287-292, 2016.

[22] R. Ivković, I. Milošević, M. Petrović, P. Spalević and S. Panić, "Image Segmentation By Sobel Edge Detection Algorithm - Mosaic Method," in Sinteza 2018 International Scientific Conference on Information Technology and Data Related Research, Belgrade, Singidunum University, Serbia, pp. 189-196, 2018.

[23] R. Ivković, M. Petrović, I. Milošević, B. Jakšić, R. Bojović, "Analysis of Different Edge Detections Algorithms Through the Bit-Plane Layers," in Sinteza 2017 - International Scientific Conference on Information Technology and Data Related Research, Belgrade, Singidunum University, Serbia, pp. 302-307, 2017. 